\title{
Solar urticaria. Case report and literature review
}

\section{Urticaria solar. Reporte de un caso y revisión de la literatura}

María Raigosa, ${ }^{1}$ Yuliana Toro, ${ }^{1}$ Jorge Sánchez $z^{1,2}$

\begin{abstract}
Background: Solar urticaria is a rare type of inducible urticaria characterized by wheal and erythema formation shortly after exposure to sunlight or to an artificial light source; its pathophysiology is not yet entirely understood. The treatment of choice, in addition to exposure avoidance, consists in antihistamine administration.

Clinical case: This is the case of a 27-year-old woman with no personal history of allergic diseases and with a 2-year history of erythema and wheals in photo-exposed areas associated with sunlight exposure for periods longer than 10 minutes. A provocation test was carried out; she was started on fexofenadine at 4-fold the standard dose (720 mg/day). Six weeks later, a new challenge was carried out without the antihistamine being discontinued; the reaction was less severe, but she continued with erythema for the first 60 minutes post-exposure. After 3 months on high-dose antihistamines, she referred marked improvement in her quality of life and tolerance to brief sunlight exposure (for less than 15 minutes).

Conclusions: Solar urticaria is a rare process but with a high impact on the patient. The use of antihistamines partially relieves symptoms and allows better tolerance to lighting expositions.
\end{abstract}

Keywords: Antihistamines; Fotosensibility; Solar urticaria; Foto-test

Este artículo debe citarse como: Raigosa M, Toro Y, Sánchez J. Urticaria solar. Reporte de un caso y revisión de la literatura. Rev Alerg Mex. 2017;64(3):371-375

${ }^{1}$ Universidad de Antioquia, IPS Universitaria, Grupo de Alergología Clínica y Experimental. Medellín, Colombia

${ }^{2}$ Fundación para el Desarrollo de las Ciencias Médicas y Biológicas. Cartagena de Indias, Colombia
Correspondencia: Jorge Sánchez. jorgem.sanchez@udea.edu.co

Recibido: 2016-07-09

Aceptado: 2016-09-07 


\section{Resumen}

Antecedentes: La urticaria solar es un tipo infrecuente de urticaria inducible que se manifiesta con erupciones y eritema poco después de la exposición a la luz del sol o de una fuente de luz artificial; su fisiopatología aún no se comprende del todo. El tratamiento de elección, además de evitar la exposición, consiste en la administración de antihistamínicos.

Caso clínico: Mujer de 27 años, sin antecedente personal de enfermedades alérgicas, con cuadro clínico de 2 años de evolución consistente en eritema y erupciones en áreas fotoexpuestas asociados con exposición a la luz del sol durante más de 10 minutos. Se realizó prueba de reto. Se decidió continuar con una dosis de fexofenadina 4 veces mayor a la convencional (720 mg/día). Seis semanas después se efectuó nuevo reto sin suspender el antihistamínico; la reacción fue menos severa, pero la paciente continuó con eritema durante los primeros 60 minutos posteriores a la exposición. Después de 3 meses con dosis altas de antihistamínicos, la paciente refirió notable mejoría en su calidad de vida y tolerancia a exposiciones cortas a luz solar (menos de 15 minutos). Conclusiones: La urticaria solar es un proceso raro pero con alto impacto en el paciente. El uso de antihistamínicos alivia parcialmente los síntomas y permite una mejor tolerancia a las exposiciones lumínicas.

Palabras clave: Antihistamínicos; Fotosensibilidad; Urticaria solar; Prueba de fotoprovocación

\section{Introducción}

La urticaria se define como una reacción caracterizada por aparición de erupciones evanescentes, eritematosas, pruriginosas y de tamaño variable. Según su duración menor o mayor a 6 semanas se ha clasificado en aguda o crónica. ${ }^{1}$ Aproximadamente $20 \%$ de los pacientes con urticaria crónica también presenta urticaria inducida por disparadores exógenos, ${ }^{2}$ ya sea físicos (presión, fricción), térmicos (frio, calor) o radiación electromagnética (radiación solar).

El diagnóstico de urticaria inducible se establece por historia clínica y examen físico, no obstante, en numerosas ocasiones es difícil identificar el factor que actúa como desencadenante, por lo que es necesario realizar una exposición controlada al estímulo sospechoso para confirmar la causalidad y establecer medidas de evitación pertinentes. ${ }^{3}$

La urticaria solar es una rara fotodermatosis que ocurre minutos a horas después de la exposición a luz solar o a una fuente de luz artificial; ${ }^{4}$ suele remitir antes de 24 horas. Si bien representa menos de $1 \%$ de todas las formas de urticaria, ${ }^{5}$ tiene un impacto negativo importante en la calidad de vida y un elevado costo económico, ${ }^{6}$ razones por las cuales el diagnóstico y manejo de esta enfermedad deben ser una prioridad para el médico tratante. A continuación presentamos un reporte de caso y la información derivada de la revisión del tema.

\section{Caso clínico}

Mujer de 27 años, sin antecedente personal de enfermedades alérgicas, con cuadro clínico de 2 años de evolución consistente en eritema y erupciones en áreas fotoexpuestas posteriores a la exposición a la luz del sol durante periodos mayores de 10 minutos. La duración de las manifestaciones solía ser menor de 24 horas; por lo general sin lesiones residuales. La paciente no indicó otros detonantes o síntomas ni antecedentes familiares similares. Antes de la consulta que aquí se refiere, la paciente había recibido varios tratamientos, entre ellos antihistamínicos $(10 \mathrm{mg} /$ día de cetirizina y $10 \mathrm{mg} /$ día de loratadina), cloroquina y fototerapia (más de 40 sesiones), sin mejoría de los síntomas.

Para identificar la causa se realizó una prueba de provocación controlada con exposición directa a luz del sol en la espalda por 25 minutos, equivalente aproximadamente a $280 \mathrm{~J}$, y posterior vigilancia durante 2 horas. En el transcurso de la exposición, la paciente presentó eritema y erupciones que persis- 
tieron después del retiro de la luz solar (Figura 1). Inmediatamente, la paciente recibió esteroide tópico y loratadina, $10 \mathrm{mg}$ iniciales y $10 \mathrm{mg}$ adicionales a los 30 minutos, con los cuales el eritema y las erupciones desaparecieron a la hora. Debido a la falta de respuesta con dosis convencionales de antihistamínicos y la demostración de una reacción inmediata con la exposición solar, se decidió una dosis de antihistamínico (fexofenadina) 4 veces mayor a la convencional ( $720 \mathrm{mg} /$ día). Seis semanas después se realizó un nuevo reto sin suspender el antihistamínico. En esa ocasión, la reacción fue menos severa, pero continuó presentándose eritema durante los primeros 60 minutos posteriores a la exposición. Después de 3 meses con dosis altas de antihistamínicos, la paciente refirió notable mejoría en su calidad de vida ya que toleraba exposiciones cortas (menores de 15 minutos) a luz solar, lo que le permitía realizar sus actividades cotidianas. Si la exposición se prolongaba, las erupciones reaparecían, pero con una severidad menor que antes del uso de antihistamínicos a dosis máxima.

\section{Discusión}

La urticaria solar es una patología poco frecuente, pero de alto impacto en la calidad de vida; adicionalmente puede dejar cicatrices con mala resolución y afectar de forma importante las relaciones del paciente en los ámbitos laboral y social. En la actualidad no existe un tratamiento que permita la remisión completa de la enfermedad, por lo que evitar la radiación social sigue siendo el manejo principal.

Las erupciones de la urticaria solar se presentan típicamente entre 1 y 15 minutos después de la exposición a la luz ultravioleta o visible. Se han reportado pocos casos de manifestaciones atípicas de urticaria solar como lesiones posteriores a 1 o 3 horas de exposición a la luz, persistencia de estas durante más de 24 horas o angioedema solar. ${ }^{7} \mathrm{La}$ gravedad de las lesiones está directamente asociada con el tiempo de exposición; si este es prolongado o en una superficie corporal amplia pueden aparecer síntomas sistémicos como mareo, somnolencia, sibilancias, disnea o colapso (anafilaxia).

La urticaria solar se presenta generalmente durante la juventud y es más frecuente en las mujeres. Sin tratamiento, la paciente descrita desarrollaba el cuadro aún con exposiciones menores de 5 minutos, las erupciones eran generalizadas incluso por exposición localizada, lo que indicaba alta severidad del cuadro.

Es importante diferenciar la urticaria solar de otras fotodermatosis como la erupción polimorfa lumínica o la porfiria eritropoyética, lo cual es relativamente fácil ya que las lesiones suelen ser eccematosas. Actualmente, la urticaria solar se clasifica en tipos I y II. El tipo I es causado por un cromóforo anormal

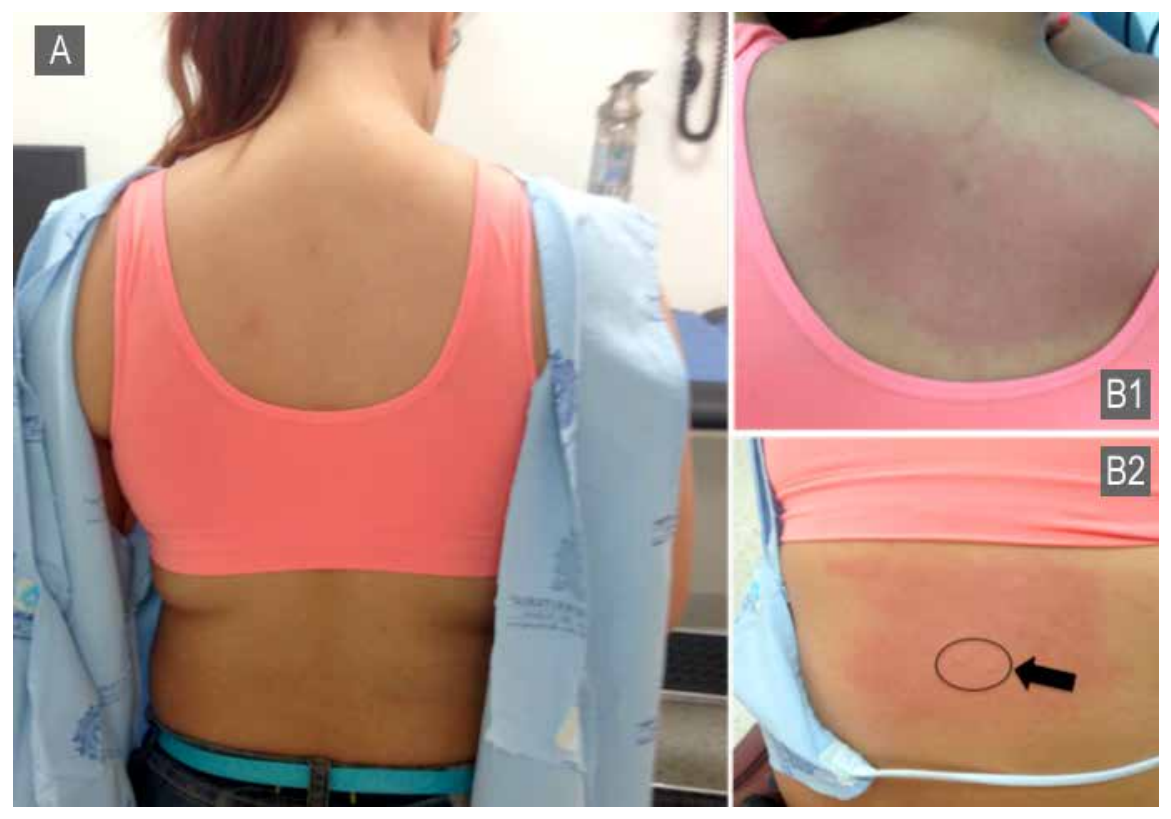

Figura 1. A) Minutos antes de la prueba de exposición solar controlada. B1) Después de 20 minutos de exposición a la luz solar se observó lesión macular notablemente eritematosa en sitios fotoexpuestos. B2) En el dorso de espalda también se formaron erupciones. 
presente en la piel de los pacientes y anticuerpos tipo IgE dirigidos contra estos. Cuando se hacen pruebas de transferencia pasiva de suero de pacientes con esta modalidad de urticaria a controles sanos, los resultados son negativos. El tipo II es causado también por anticuerpos tipo IgE, pero contra cromóforos normales, por lo tanto, los resultados son positivos en las pruebas de tranferencia pasiva de suero de pacientes con urticaria a controles sanos. ${ }^{8}$ Los cromóforos se activan durante la irradiación solar, convirtiéndose en fotoalérgenos que son reconocidos por la IgE. En la paciente no fue posible aclarar el mecanismo fisiopatológico ya que los exámenes necesarios no están disponibles en nuestro centro.

Los pacientes con urticaria solar pueden no identificar correctamente el detonante e indicar erróneamente que la enfermedad se debe a procesos como la exposición al calor o al ejercicio, lo que lleva a restricciones innecesarias. Para confirmar la urticaria solar se debe realizar una prueba de fotoprovocación, la cual no cuenta con guías protocolizadas para su realización. La prueba de fotoprovocación consiste en administrar altas dosis luminosas en forma repetida sobre una superficie cutánea de 20 a $25 \mathrm{~cm}^{2}$; puede realizarse con una fuente emisora de rayos UVB (280 a $320 \mathrm{~nm})$, UVA ( 320 a $400 \mathrm{~nm}$ ) o una fuente emisora de luz visible (400 a $600 \mathrm{~nm}$ ), que se reproduce con un proyector de diapositivas o directamente con la luz solar.

En la literatura existen reportes de casos de pacientes con urticaria solar que han tenido resultados negativos en las pruebas de fotoprovocación cuando estas se realizan con espectros independientes de luz, ${ }^{1}$ lo cual nos motivó a realizar una prueba directamente con luz solar en nuestra paciente. Lo anterior sugiere que algunos pacientes requieren una combinación de varios espectros lumínicos para que se desencadenen las lesiones. Adicionalmente, la provocación solar nos permitió hacer el diagnóstico y evaluar la respuesta al tratamiento con un antihistamínico de acuerdo con el tiempo de tolerancia a una nueva exposición solar y la severidad de los síntomas.

Las medidas de evitación y el uso de protectores antisolares permiten un control de la enfermedad, sin embargo, estas medidas implican importantes restricciones y cambios en el estilo de vida que dificultan su continua realización. El uso de antihistamínicos ha sido ampliamente recomendado en pacientes con urticaria, incluyendo la solar; se recomienda aumentar hasta 4 veces la dosis del antihistamínico si la respuesta es insuficiente con una dosis convencional. ${ }^{9}$ Aun cuando no se alcanzó un control completo, en nuestra paciente se prolongó la tolerancia a la luz del sol, con mejoría de la calidad de vida y menor limitación en las actividades cotidianas.

El omalizumab, un anticuerpo monoclonal antiIgE, se ha descrito como alternativa terapéutica en pacientes con urticaria crónica resistente al tratamiento con antihistamínicos. En la literatura existen reportes de casos y series de casos con urticaria solar refractaria en los que se registran buenos resultados con este medicamento. ${ }^{10} \mathrm{Si}$ bien hasta el momento del presente informe la paciente no había recibido omalizumab, consideramos puede ser una opción de acuerdo con la evolución de los síntomas.

En conclusión, un diagnóstico adecuado con las pruebas de fotoprovocación permite realizar una evitación específica del verdadero agente causal de los síntomas en la urticaria. El uso de antihistamínicos en dosis altas ayuda a disminuir la gravedad del cuadro y permite mayor grado tolerancia a la luz solar.

\section{Referencias}

1. Zuberbier T, Aberer W, Asero R, Bindslev-Jensen C, Brzoza Z, Canonica GW et al. The EAACI/GA(2) LEN/EDF/WAO guideline for the definition, classification, diagnosis, and management of urticaria: The 2013 revision and update. Allergy. 2014;69(7):868-887. DOI: http://dx.doi.org/10.1111/all.12313

2. Trevisonno J, Balram B, Netchiporouk E, Ben-Shoshan M. Physical urticaria: Review on classification, triggers and management with special focus on prevalence including a meta-analysis. Postgrad Med. 2015;127(6):565-570. DOI: http://dx.doi.org/10.1080/00325481.2015.1045817

3. Bernstein JA, Lang DM, Khan DA, Craig T, Dreyfus D, Hsieh F, et al. The diagnosis and management of acute and chronic urticaria: 2014 update. J Allergy Clin Immunol. 2014;133(5):1270-1277. DOI: http:// dx.doi.org/10.1016/j.jaci.2014.02.036 
Raigosa M et al. Urticaria solar. Reporte de caso

4. Nitiyarom R, Wongpraparut C. Hydroa vacciniforme and solar urticaria. Dermatol Clin. 2014;32(3):345353, DOI: http://dx.doi.org/10.1016/j.det.2014.03.013

5. Amaya D, Sánchez A, Sánchez J. Inducible urticaria: Case series and literature review. Biomedica. 2016;36(1):10-21. DOI: http://dx.doi.org/10.7705/biomedica.v36i1.2678

6. Scott CB, Moloney MF. Physical urticaria: A common misdiagnosis. Nurse Pract. 1996;21(11):42-46, 9-54 passim; quiz 9-61.

7. Fesq H, Ring J, Abeck D. Management of polymorphous light eruption: Clinical course, pathogenesis, diagnosis and intervention. Am J Clin Dermatol. 2003;4(6):399-406.

8. Pérez-Ferriols A, Aguilera J, Aguilera P, De-Argila D, Barnadas MA, de-Cabo X, et al. Determination of minimal erythema dose and anomalous reactions to UVA radiation by skin phototype. Actas Dermosifiliogr. 2014;105(8):780-788. DOI: http://dx.doi.org/10.1016/j.ad.2014.05.001

9. Magerl M, Altrichter S, Borzova E, Giménez-Arnau A, Grattan CE, Lawloe F, et al. The definition, diagnostic testing, and management of chronic inducible urticarias-The EAACI/GA(2) LEN/EDF/UNEV consensus recommendations 2016 update and revision. Allergy. 2016;71(6):780-802. DOI: http://dx.doi. org/10.1111/all.12884

10. Baliu-Piqué $\mathrm{C}$, Aguilera-Peiró P. Three cases of solar urticaria successfully treated with omalizumab. J Eur Acad Dermatol Venereol. 2016;30(4):704-706. DOI: http://dx.doi.org/10.1111/jdv.13001 